\title{
Combined Laryngospasm and Bronchospasm during Procedural Anesthesia
}

\author{
Zachary Daniels ${ }^{1}$, Andrew C Jenzer ${ }^{2 *}$ and Jeremy J Hanlon ${ }^{3}$ \\ ${ }^{1}$ Department of Oral and Maxillofacial Surgery, Womack Army Medical Center, USA \\ ${ }^{2}$ Department of Oral and Maxillofacial Surgery, Eisenhower Army Medical Center, USA \\ ${ }^{3}$ Department of Oral and Maxillofacial Surgery, Womack Army Medical Center, USA
}

Submission: November 04, 2019; Published: November 15, 2019

*Corresponding author: Andrew C Jenzer, Department of Oral and Maxillofacial Surgery, Eisenhower Army Medical Center, Fort Gordon, USA

\begin{abstract}
Background and Overview: Laryngospasm and bronchospasm are known complications of anesthesia, and of special concern with an open airway during procedural sedation, especially during oral surgery. The authors report on a case of combined laryngospasm and bronchospasm with a patient with no preoperative risk factors.

Case Description: A healthy 20-year-old male presented for routine wisdom teeth extraction and frenectomy under deep sedation at a hospital-based Army oral \& maxillofacial surgery residency training program. The patient had no significant past medical history, allergies, or pre-operative risk factors suggestive for pulmonary concerns such a smoking, asthma, seasonal allergies, or a history of anesthetic complications. After starting the sedation and while providing local anesthesia, the patient desaturated with the surgeon-anesthetist noting stridor leading to silence on the precordial stethoscope. Help was called for after diagnosing a laryngospasm. This was broken with positive pressure while preparing for general anesthesia level intervention. The patient still did not regain saturation and was wheezing significantly on auscultation with markedly decreased compliance and a bronchospasm was identified. The patient was successfully treated with intravenous epinephrine, regained normal saturation, the procedure was aborted, and the patient was recovered to be discharged with plans for reappointment in the main operating room to proceed under general anesthesia.

Conclusion and Practical Implications: Reviewing possible causes for laryngospasm and bronchospasm, this was an abnormal presentation as surgery had not yet begun, with minimal secretions and no irrigation in the airway, the usual culprit of these complications. Laryngospasms and bronchospasms are known complications with sedations and anesthesia. There was no obvious trigger for these events in this case and no indication in the pre-operative assessment that this patient would have a reactive airway or be at an increased risk for complications. However, like for every sedation and anesthesia case, a full emergency kit was in the room and was utilized following an emergency response plan. The critical points of this event were diagnosing first a laryngospasm and treating it and then after breaking it then diagnosing a bronchospasm and treating that.
\end{abstract}

Keywords: Laryngospasm; Bronchospasm; Anesthesia; Procedural sedation

\section{Case Report}

The following presents a case of a male active duty service member without any significant past medical history or allergies and no pulmonary risk factors who experienced a combined laryngospasm and bronchospasm at the beginning of a procedural sedation.

A healthy 20-year-old male presented for routine wisdom teeth extraction and frenectomy under deep sedation at a hospital-based army Oral \& maxillofacial surgery residency training program. The patient had no significant past medical history, allergies, or pre-operative risk factors suggestive for pulmonary concerns such a smoking, asthma, seasonal allergies, or a history of anesthetic complications. Prior to beginning the procedure, the resident surgeon-anesthetist presented their case to the attending surgeon and obtained permission to initiate deep sedation and surgery. The emergency medications and supply kit were brought into the procedure room. Consents, medication reconciliation, and time out protocol were completed per institution protocol. The patient reported he was very nervous for surgery, especially for the intravenous access and became visibly diaphoretic before starting. A cold compress was placed to forehead and the patient was positioned to his comfort. The patient then reported nausea. An intravenous catheter was placed, and the patient was administered Zofran 4mg, supplemental oxygen was applied at 3LPM via nasal cannula. Bluetooth precordial stethoscope was connected and remained 
on for the duration of the case as an additional airway monitor. At this time, the patient reported resolution of diaphoresis and nausea, so a final time-out was completed. Medications consisting of $5 \mathrm{mg}$ of midazolam and $100 \mathrm{mcg}$ of fentanyl were slowly and sequentially administered intravenously without incident. Next, $2 \mathrm{~g}$ of cefazolin was reconstituted and added to the bag of $500 \mathrm{~mL}$ lactated ringers for slow infusion during the procedure. The resident surgeon-anesthetist talked to patient, maintaining verbal communication to continually assess the patient while positioning for the procedure. The patient was still talking to team coherently at this point and was administered $40 \mathrm{mg}$ of propofol prior to local anesthetic injections. The patient's eyes were noted to close and a head wrap placed. Next, 30mg of Toradol and 8mg of Decadron were administered intravenously without incident.

The local anesthetic phase of the procedure began by injecting the right side of the oral cavity (weight appropriate doses of $2 \%$ lidocaine with 1:100,000 epinephrine and 0.5\% Marcaine with $1: 200,000$ epinephrine). It was then noted that the patient began to desaturate from a Sp02 in the high 90s to the mid-80s. The patient was jaw thrusted with Larsen maneuver and his SpO2 held in mid 80s briefly and began to rapidly normalize, at which point more local anesthetic was administered. While injecting the left side of the oral cavity, the provider auscultated squeaky/ whistling breathing thru the precordial stethoscope and the patient's saturation dropped precipitously down to 59\% Sp02. During the desaturation, local anesthetic administration was terminated, Larsen maneuver resumed, and the supplemental oxygen was increased from 3LPM to 8LPM. The patient was aggressively jaw thrusted and his tongue clamped with a Kelly and pulled to maximal extension with no improvement in saturation or breath sounds. At this time the recorder was dispatched to obtain immediate help from attending surgeons. The attending surgeon arrived in under one minute by which time the patient's saturation had climbed back to mid-80s, but breath sounds were still not normal but less stridor and transmitted breath sounds. The patient was monitored by the team while maintaining the above measures, but within one minute the patient rapidly desaturated into the $30 \mathrm{~s}$ and his lips were noted to be blue and his skin dusky bradycardia noted with junctional rhythm. Immediately bag-valve mask ventilation was initiated increasing supplemental oxygen to the maximum flow of 15LPM with the arrival of additional staff surgeons. At this point, the precordial stethoscope and traditional stethoscope auscultation were absent of any sounds and almost no air was provided by bag-valve mask (BVM) with the main effect of inflating the patient's cheeks - laryngospasm was identified and communicated among group of providers. The patient's saturation stops reading and the waveform dropped off the monitor at this time. One surgeon continued to provide two hand jaw thrust while another continued to attempt to break the laryngospasm with positive pressure, as other members drew up succinylcholine and prepared to intubate.

At this time a Code Blue was about to be called for additional assistance. Before this could be done or any medications could be pushed, the laryngospasm was broken by positive pressure BVM. The precordial sounds went from complete silence to a squeaky gasp and then to normal airflow. However, the patient's saturation was still not reading, despite staff efforts to address the monitors, the patient's lips were still blue, and bagging him was unusually difficult. The patient was auscultated again and noted significant wheezing and minimal airflow to bilateral lungs - a bronchospasm was identified. The team attempted to administer Albuterol thru syringe to BVM but there was no attachment connection, so multiple applications of multiple puffs of Albuterol was administered into mouth and delivered with positive pressure. Saturation then came back on screen in 30s although no clinical improvement was noted - the patient was still bradycardic but with bigeminy. Then, $100 \mathrm{mcg}$ of epinephrine was administered via rapid intravenous push with near-immediate spike in vitals, including SpO2 increasing into 70s with easier BVM. Additional albuterol was administered as the patient's respiratory status normalized and his saturation increased to the upper 90s. BVM was maintained with a good seal and the rate was decreased from roughly $30 \mathrm{bpm}$ to $10 \mathrm{bpm}$. Over the next several minutes the patient began to initiate breaths on his own and active bagging was discontinued. After several minutes, the patient was trialed on nasal cannula at 5LPM, then decreased to 3LPM of supplemental oxygen. The patient was then found to be electrically and hemodynamically normal. After 10 minutes, nasal cannula was discontinued and patient-maintained saturation on room air of upper 90s for 30 minutes before being discharged after meeting established criteria, and his escort being informed of the complication. The patient was carefully followed up in clinic with no sequelae and the case was later completed in the main operating room under full general anesthesia with no complications.

\section{Discussion}

Laryngospasm is a known, and feared, complication of anesthesia. It occurs with an incidence of .8\% to $25 \%[1,2]$. With vagal stimulation of the superior laryngeal nerve, the vocal cords adduct and close with air movement becoming obstructed [3]. This reflex exists to protect the pulmonary system from aspiration and is pronounced during stage II of anesthesia (of the Guedel classification) when the airway is at its most reactive point [4]. The reflex is exacerbated by objects, foreign debris, and secretions in the airway. Clinical signs and symptoms include high-pitched squeaking sounds (stridor), sudden inability to ventilate, and a decrease in oxygen saturation. Treatment includes removal of irritating factors, initiating a painful stimulus commonly through jaw thrust with Larson's maneuver and/or sternal rub, positivepressure ventilation, small doses of a rapid onset neuromuscular drug (typically succinylcholine), and deepening or lightening of the anesthetic plane [3-5]. Bronchospasm is a second known, and feared, complication of anesthesia with incidence is reported at $1.7 \%$ and higher $[1,6,7]$. Asthmatics exhibit a slightly higher incidence of bronchospasm, with other risk factors including history of eczema, smoking, younger age, and current or recent 
upper respiratory tract infection [6]. Causes include anaphylaxis, excessive bronchial responsiveness, neuromuscular drugs, tracheal irritation, or other drug reactions. Clinical signs and symptoms include wheezing needs a period. Treatment includes inhaled $\beta 2$-selective agonists, discontinuing any noxious inhaled agents, deepening the anesthetic with drugs such as propofol and/or ketamine, and intravenous epinephrine, with slower acting therapies to include anticholinergics and steroids [6-8].

Reviewing possible causes for laryngospasm and bronchospasm, this case represented an abnormal presentation as surgery had not yet begun, and minimal secretions actively controlled with suctioning. The patient possessed no pre-operative risk factors that indicate a reactive airway such as asthma, smoking, seasonal allergies, or previous anesthetic complications. This may have been a possible Type 1 hypersensitivity reaction to Ancef and/or Toradol although the patient denied having any drug allergies, although it could have just as plausibly occurred due to saliva falling on the vocal cords or any other noxious stimuli triggering this exaggerated reaction. The patient exhibited hyper-responsiveness throughout the airway and represents a very dangerous situation for procedural sedation. A key point in this case and the subsequent successful management of these complications was having a full emergency kit in the room and a trained and followed emergency response plan. The critical points of this event were diagnosis and treatment of first a laryngospasm followed by bronchospasm. Like any emergency, there was an element of chaos which slows the response but mitigated through teamwork and practiced emergency response training. This case serves as a stark reminder that despite all efforts at pre-screening and risk stratification, adverse events can occur at even the most seemingly safe portions of a procedural sedation and providers must have the planning, trading, and skills to address such events.

\section{Disclaimer}

The views expressed herein are those of the authors and do not reflect the official policy of the Department of the Army, Department of Defense, or the U.S. government.

\section{References}

1. Olsson GL, Hallen B (1984) Laryngospasm during anesthesia: A computer-aided incidence study in 136,929 patients. Acta Anaesthesiol Scand 28(5): 567-75.

2. Burgoyne LL, Anghelescu DL (2008) Intervention steps for treating laryngospasm in pediatric patients. Paediatr Anaesth 18(4): 297-302.

3. Pardo, Manuel, Miller, Ronald (2017) Basics of Anesthesia. Elsevier 31: 524-536.

4. Artime, Carlos, Hagberd, Carin (2019) Miller's Anesthesia. Elsevier, 2019; 44: 1373-1412.

5. AlAlami AA, Ayoub CM, Baraka AS (2008) Laryngospasm: review of different prevention and treatment modalities. Paediatr Anaesth 18(4): 281-288.

6. Benca, Joan (2018) Complications in Anesthesia. Elsevier 134: 527 529.

7. Woods BD, Sladen RN (2009) Perioperative considerations for the patient with asthma and bronchospasm. Br J Anaesth 103(1): 57-65.

8. Liccardi G, Salzillo A, Sofia M, D'Amato M, D’Amato G (2012) Bronchial Asthma. Curr Opin Anesthesiol 25(1): 30-37.

9. Miloro M, Kolokythas A (2011) Management of Complications in Oral and Maxillofacial Surgery. Wiley Publishing 2: 2-24.

\section{Your next submission with Juniper Publishers} will reach you the below assets

- Quality Editorial service

- Swift Peer Review

- Reprints availability

- E-prints Service

- Manuscript Podcast for convenient understanding

- Global attainment for your research

- Manuscript accessibility in different formats

( Pdf, E-pub, Full Text, Audio)

- Unceasing customer service

Track the below URL for one-step submission

https://juniperpublishers.com/online-submission.php 